\title{
Different Phytohormonal Responses on Satsuma Mandarin (Citrus unshiu) Leaves Infected with Host-Compatible or Host-Incompatible Elsinoë fawcettii
}

\author{
Kihye Shin (1D *, Dilli Prasad Paudyal", Seong Chan Lee, and Jae Wook Hyun* \\ Citrus Research Institute, National Institute of Horticultural and Herbal Science, Rural Development Administration, \\ Jeju 63607, Korea \\ 'Current address: miniPCR, Amplyus LLC, Arlington, MA 02474, USA
}

(Received on December 13, 2020; Revised on April 5, 2021; Accepted on April 23, 2021)

Citrus scab, caused by the fungal pathogen Elsinö fawcettii, is one of the most important fungal diseases affecting Citrus spp. Citrus scab affects young tissues, including the leaves, twigs, and fruits, and produces severe fruit blemishes that reduce the market value of fresh fruits. To study the molecular responses of satsuma mandarin (C. unshiu) to $E$. fawcettii, plant hormone-related gene expression was analyzed in response to host-compatible (SM16-1) and host-incompatible (DAR70024) isolates. In the early phase of infection by E. fawcettii, jasmonic acid- and salicylic acid-related gene expression was induced in response to infection with the compatible isolate. However, as symptoms advanced during the late phase of the infection, the jasmonic acid- and salicylic acid-related gene expression was downregulated. The gene expression patterns were compared between compatible and incompatible interactions. As scabs were accompanied by altered tissue growth surrounding the infection site, we conducted

*Co-corresponding authors.

K. Shin

Phone) +82-64-730-4183, FAX) +82-64-733-9564

E-mail) kihyeshin@korea.kr

J. W. Hyun

Phone) +82-64-730-4101, FAX) +82-64-733-9564

E-mail)hyunjaewook@korea.kr

ORCID

Kihye Shin

https://orcid.org/0000-0001-8044-4530

Handling Editor : Jeum Kyu Hong

(c) This is an Open Access article distributed under the terms of the Creative Commons Attribution Non-Commercial License (http:// creativecommons.org/licenses/by-nc/4.0) which permits unrestricted noncommercial use, distribution, and reproduction in any medium, provided the original work is properly cited.

Articles can be freely viewed online at www.ppjonline.org. gibberellic acid- and abscisic acid-related gene expression analysis and assessed the content of these acids during scab symptom development. Our results showed that gibberellic and abscisic acid-related gene expression and hormonal changes were reduced and induced in response to the infection, respectively. Accordingly, we propose that jasmonic and salicylic acids play a role in the early response to citrus scab, whereas gibberellic and abscisic acids participate in symptom development.

Keywords : citrus scab, Citrus unshiu, Elsinoë fawcettii, fungal pathogen, satsuma mandarin

Citrus scab, one of the most destructive diseases in Citrus spp., and has been reported in several citrus-producing countries worldwide, especially under humid cultivation conditions. The associated pathogen, Elsinoë fawcettii Bitancourt and Jenkins, affects the young tissues of fruits, leaves, and twigs of various susceptible cultivars of citrus, lemons, grapefruit, tangerines, and their hybrids (Chung, 2011; Gopal et al., 2014). Scab infection leads to abnormal and reduced growth, and even dieback and fruit drop, and causing serious fruit blemishes that reduce the external quality and market value of citrus fruits, resulting in heavy economic losses to citrus producers.

Scab lesions are visible on the leaves at 3 days post inoculation (dpi) and on the fruits at 7 dpi (Paudyal et al., 2017). The lesions appear as tiny dots, and as the host tissues expand, the infected areas become elevated and form erumpent scab pustules comprising fungal and host tissues. The affected leaves may develop lesions with warty or protuberant pustules emerging from one side of the leaf and a correspondingly depressed area on the opposite side. When the pathogen infects young fruits, groups of light brown, 
raised, rounded, and warty scabs appear on the rind surface. Extensive scabby areas may form individual pustules, which merge into one another as they age (Chung, 2011; Gopal et al., 2014). These cone-like raised structures are commonly observed in other fungal diseases, in not only citrus but also other plants.

Although the physiological aspects and infection mechanism have been well studied (Chung, 2011; Gopal et al., 2014; Paudyal and Hyun, 2015; Paudyal et al., 2017), the exact mechanism of plant-pathogen compatibility and the reasons for the abnormal cell growth in E. fawcettiiinfected leaves are still unknown. For molecular breeding and effective disease control, understanding the molecular mechanism underlying the infection is imperative. Therefore, using host-compatible (SM16-1) and host-incompatible (DAR70024) E. fawcettii isolates, we investigated the hormonal responses of plants with citrus scab to understand the mechanism underlying infection and symptom development (Timmer et al., 1996; Hyun et al., 2001, 2009).

Plant defense against pathogens depends on the interplay of different signaling mechanisms, such as those mediated by the hormones salicylic acid (SA) and jasmonic acid (JA) (Berens et al., 2017; Shigenaga and Argueso, 2016). In addition to these well-characterized hormone pathways, other biochemical pathways mediated by hormones such as abscisic acid (ABA), gibberellic acid (GA), brassinosteroids, and auxins are identified as important players in plant defense against necrotrophic fungi. JA plays an essential role in plant defense responses against necrotrophic and hemibiotrophic pathogens, especially fungi (Antico et al., 2012; Berens et al., 2017). Mutants of the biosynthetic and signaling genes of JA show increased susceptibility to various fungi; furthermore, exogenous application of methylJA induces resistance to plants against fungal pathogens (Oliveira et al., 2015). Moreover, SA induces plant defense responses against biotrophic pathogens and leads to the production of antimicrobial molecules, such as pathogenesis-related proteins and phytoalexins, to induce hypersensitive responses. In addition, $\mathrm{ABA}$ regulates several aspects of plant development, such as seed dormancy and germination, as well as abiotic and biotic stress responses (Finkelstein et al., 2002; Vishwakarma et al., 2017). Moreover, the perception and signaling pathways of ABA have been well studied at the molecular level (Park et al., 2009). However, the role of $\mathrm{ABA}$ in plant defense against pathogens varies depending on host-pathogen diversity, and it seems to play different roles at different time points after infection (Berens et al., 2017; Ton et al., 2009).

In this study, we aimed to identify different hormonal regulations in compatible (SM16-1) isolate- and incompat- ible (DAR70024) isolate-inoculated satsuma mandarin leaves at the early infection stage and symptom development stage. Hence, to examine hormonal regulation, we assessed the expression of JA-associated genes: COI1 (coronatine insensitive 1) and JARl (jasmonate resistant 1), SA-associated genes: NPR1 (nonexpressor of PR1 genes 1) and $E D S 16$ (enhanced disease susceptibility to Erysiphe oronti 16), ABA-associated genes: NCED3 (9-cis-epoxycarotenoid dioxygenase 3), and $A B F 3$ (abscisic acid responsive elements-binding factor3), GA-associated genes: GA20ox2 (gibberellin 20 oxidase 2) and GA2ox2 (gibberellin 2-oxidase 2) (Abdelkareem et al., 2017; Dewdney et al., 2000; Fan and Dong, 2002; Rieu et al., 2008; Staswick et al., 2002; Sun, 2008; Sussmilch et al., 2017; Yoshida et al., 2010). Furthermore, we analyzed the hormone contents during infection and symptom development to understand how citrus plants responds to E. fawcettii infection and how the symptoms develop. Although the signaling of hormonal regulation during fungal pathogen infection has been reported in Arabidopsis and other model plant species, our study is the first to investigate these phenomena in satsuma mandarin leaves inoculated with compatible scab isolate and incompatible scab isolates.

\section{Materials and Methods}

Fungal isolate preparation. E. fawcettii 16-1, a Florida broad host range pathotype, and E. fawcettii DAR70024 of Tryon's pathotype, which have been deposited at the Korea Agricultural Culture Collection (KACC45780), were used in this study (Hyun et al., 2001). To produce conidia, the shaking method was used, as previously reported (Hyun et al., 2015). Dried and preserved mycelia were cut into small pieces and cultured on potato dextrose agar (PDA) (BD, East Rutherford, NJ, USA) at $25^{\circ} \mathrm{C}$ for 2 weeks. The mycelial mat that developed on PDA was transferred into a mortar without media and homogenized. The homogenized mycelia were then transferred into $50 \mathrm{ml}$ of sterilized water and cultured for sporulation at $25^{\circ} \mathrm{C}$ for $24 \mathrm{~h}$ in a shaker under dark conditions. The conidial suspension was filtered through two layers of cheesecloth, and then quantified using a hemocytometer (Paul Marienfeld GmbH \& Co., KG, Lauda-Königshofen, Germany).

Fungal inoculation. Satsuma mandarin (Citrus unshiu Marc) plants aged 4-5 years were used for fungal inoculation. The plants were grown in a growth chamber at $25^{\circ} \mathrm{C}$ under long-day conditions ( $16 \mathrm{~h} \mathrm{light} / 8 \mathrm{~h}$ dark). These plants were pruned to stimulate the production of uniform flushes of new leaves. Subsequently, the light condition 
was changed to $24 \mathrm{~h}$ of light for gene expression analysis. For each E. fawcettii isolate (SM16-1 and DAR70024), a suspension with $10^{6}$ conidia $/ \mathrm{ml}$ was sprayed on one-fifth of the mature leaves. After inoculation, the leaves were subjected to moist conditions using plastic bag covers for 2 days.

Scanning electron microscopy. Scanning electron microscopy; S-3500 N (Hitachi Corp., Tokyo, Japan) was performed to observe citrus scab symptoms on satsuma mandarin leaves. The inoculated leaves were harvested and cut into approximately $0.5 \mathrm{~cm} \times 0.5 \mathrm{~cm}$ pieces. The leaves were fixed in Karnovsky's fixative solution containing 2\% glutaraldehyde (Sigma-Aldrich, St. Louis, MO, USA) and $2.5 \%$ paraformaldehyde (Sigma-Aldrich) in $0.05 \mathrm{M}$ sodium cacodylate, which was prepared by mixing cacodylic acid (Sigma-Aldrich) and a buffer of $\mathrm{pH} 7.2$ and incubating the mixture for $2 \mathrm{~h}$ at $25^{\circ} \mathrm{C}$. Next, the samples were washed twice for $20 \mathrm{~min}$ each with $0.05 \mathrm{M}$ sodium cacodylate buffer and fixed with a second fixative, $1 \%$ osmium tetroxide (Sigma-Aldrich), for $2 \mathrm{~h}$. The samples were dehydrated using an ethanol series of $10 \%, 30 \%, 50 \%, 70 \%, 80 \%$, $90 \%$, and $95 \%$ for 15 min each, followed by dehydration with $100 \%$ ethanol for $15 \mathrm{~min}$ three times. Finally, critical dehydration was performed using a chemical dehydrant, hexamethyldisilazane (Merck \& Co., Inc., Kenilworth, NG, USA). These specimens were mounted on aluminum stubs, and when required, the specimens were sputter coated with evaporated platinum for $50 \mathrm{~min}$ in vacuum using an E-1030 ion sputter coater (Hitachi Corp.).

Gene expression analysis. Total RNA from the inoculated leaves was extracted using the Plant RNeasy kit (Qiagen, Venlo, The Netherlands) in the automated system QIAcube (Qiagen) according to the manufacturer's instructions. One microgram of RNA was reverse transcribed using
Invitrogen Superscript IV (Thermo Fisher, Waltham, MA, USA). Thereafter, 10-fold diluted cDNA was subjected to quantitative reverse-transcription polymerase chain reaction (qRT-PCR) using the primers listed in Table 1. The qRT-PCR analysis was performed using CFX96 Touch (Bio-Rad, Hercules, CA, USA) and the Power SYBR Green PCR Master Mix (Thermo Fisher). The primers used for qRT-PCR were designed based on the sequence obtained from citrusgenome.jp (Shimizu et al., 2017). The primers used are listed in Table 1. As the internal standard, CitGAPDH or CitEF1a was selected from previous studies (Calvalho et al., 2010; Mafra et al., 2012); we tested the stability in our experimental condition. CitGAPDH or CitEFla showed similar expression level (data not shown) and was used randomly in different experiment. The reactions were performed in triplicates per run, and the relative expression of each gene was determined after normalizing the expression to the expression level of $C i t G A P D H$ or CitEFla. The comparative $2^{-\Delta \Delta C t}$ method was used to evaluate the relative quantities of each amplified product in the samples, following a previously described procedure (Livak and Schmittgen, 2001).

Sample preparation for phytohormone analysis. Fresh samples $(50 \mathrm{mg})$ were frozen in liquid nitrogen and ground into a powder, which was extracted with $1 \mathrm{ml}$ of isopropanol: $\mathrm{H}_{2} \mathrm{O}: \mathrm{HCl}(2: 1: 0.002, \mathrm{v} / \mathrm{v} / \mathrm{v})$ using a vortex for $20 \mathrm{~min}$. Dichloromethane $(1 \mathrm{ml})$ was then added, and the mixture was centrifuged at $15,000 \mathrm{rpm}$ for $10 \mathrm{~min}$ for fractionation. After fractionation, two phases were obtained, where the dichloromethane fraction was eluted through a $\mathrm{C} 18$ cartridge using methanol. The filtered sample was concentrated and re-dissolved in $25 \mu \mathrm{l}$ of methanol.

Phytohormone analysis. For analysis, phytohormone extracts were injected into the Shimadzu LCMS-2020 (Shi-

Table 1. Primers used in this study

\begin{tabular}{lllc}
\hline Name & \multicolumn{1}{c}{ Forward primer (5'-3') } & \multicolumn{1}{c}{ Reverse primer (5'-3') } & Reference \\
\hline CitGAPDH & GGAAGGTCAAGATCGGAATCAA & CGTCCCTCTGCAAGATGACTCT & Carvalho et al. (2010) \\
CitEF1 $\alpha$ & TCAGGCAAGGAGCTTGAGAAG & GGCTTGGTGGGAATCATCTTAA & Carvalho et al. (2010) \\
CitCOI1 & GCGGCGATGTTTAATTGAT & GAGATCCGAATCCCTGACAA & In this study \\
CitJAR1 & GACGCGGAGAGAATTCAAAG & AGGCCGAGATTTTGCAAGTA & In this study \\
Cit $N$ PR1 & TGTGCGGTAAAGCTTGTGAG & CAATGTGTTGTGGCAAGGTC & In this study \\
CitEDS16 & GCTCAGCACCAACTTCTTCC & GTTGGGAAAGACAACGGAAA & In this study \\
CitGA2ox2 & AAGGAGTGACTCGTGCTTCA & TGGATTTGGAGGCCAGATGT & In this study \\
CitGA20ox2 & GATCTTGCTCTTGGCACTGG & CTAGGGCATCATCACGAGGT & In this study \\
Cit $N C E D 3$ & CGTTCATAGGCGCGAAAGAT & TGCAAGCATCCCTCTGGTAA & In this study \\
CitABF4 & ATCAAATGCTCCTGGTGGGA & CCAGCCTGAACTAAGTTGCC & In this study \\
\hline
\end{tabular}


madzu Corporation, Kyoto, Japan) system coupled with the YMC-Triart C18 ExRS column $(100 \mathrm{~mm} \times 2.0 \mathrm{~mm}$; internal diameter, $8 \mathrm{~nm}$; particle size, $1.9 \mu \mathrm{m})$. Thereafter, 10 $\mu \mathrm{L}$ of the extract was loaded into the system using a mobile phase comprising (A) water with $0.2 \%$ formic acid and (B) acetonitrile with $0.2 \%$ formic acid. The flow rate of the mobile phase was set at $0.25 \mathrm{ml} / \mathrm{min}$, and gradient elution was applied. For conditioning, 100\% solvent was eluted for $1 \mathrm{~min}$, followed by $30 \% \mathrm{~B}$ for $3 \mathrm{~min}$ and then $60 \% \mathrm{~B}$ for $17 \mathrm{~min}$. For mass spectrometry analysis, the following conditions were used: nebulizing gas flow rate, $1.5 \mathrm{l} / \mathrm{min}$; drying gas flow rate, $15 \mathrm{l} / \mathrm{min}$; scan mode, negative; mass analysis range, $120-550 \mathrm{~m} / \mathrm{z}$; and scan speed, $883 \mathrm{u} / \mathrm{s}$.

\section{Results}

Different disease symptoms caused by compatible or incompatible citrus scab pathogen on satsuma mandarin leaves. To investigate scab disease development, two different pathotypes of citrus scab isolates, SM16-1 and DAR70024, which are compatible and incompatible with satsuma mandarin, respectively, were used in this study (Table 2) (Hyun et al., 2009). At 6 dpi, typical symptoms of citrus scab, such as irregular scabby and wartlike outgrowths, were observed in the leaves inoculated with SM16-1. However, in the leaves inoculated with DAR70024, such scabby outgrowths were not observed, but several dark spots of unknown origin were observed (Fig. 1A-C).

To better understand the development of citrus scab symptoms, we observed the morphological differences between leaves inoculated with SM16-1 and those inoculated with DAR70024 by scanning electron microscopy. In a previous study, the germination of conidia was observed at 1 dpi in SM16-1-inoculated samples (Paudyal and Hyun, 2015). Thus, we compared different features of infection sites at $1 \mathrm{dpi}$ and observed degraded cuticle layers in the infection sites on the leaves inoculated with each of SM161 and DAR70024 isolate. However, the degradation pattern differed between the isolates. The SM16-1-inoculated leaves showed a bottle-necked groove at the center of the infection site, where the necrotic area was enveloped and partially covered by enlarged and rapidly dividing cells, whereas the DAR70024-inoculated leaves showed smaller and shallower necrotic regions. Moreover, the necrotic region surrounding the cells was not visibly changed (Fig. 1D). This result showed that the initial symptoms, including the necrotic region and surrounding cell alteration at the infection site, were different at $1 \mathrm{dpi}$. Thus, plants may endogenously respond differently to each isolate as early
A

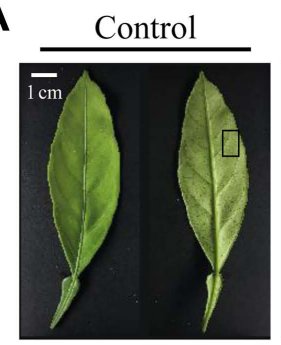

B

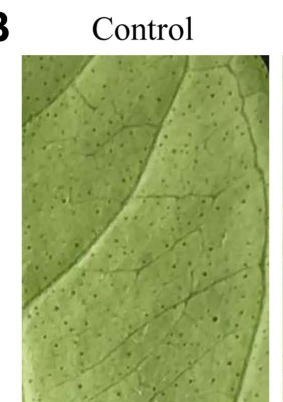

C

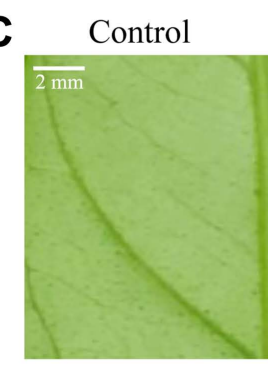

D Control

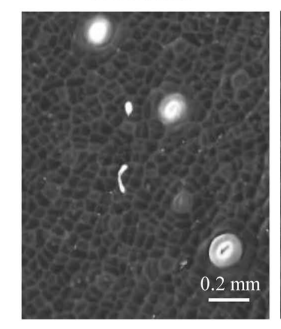

E
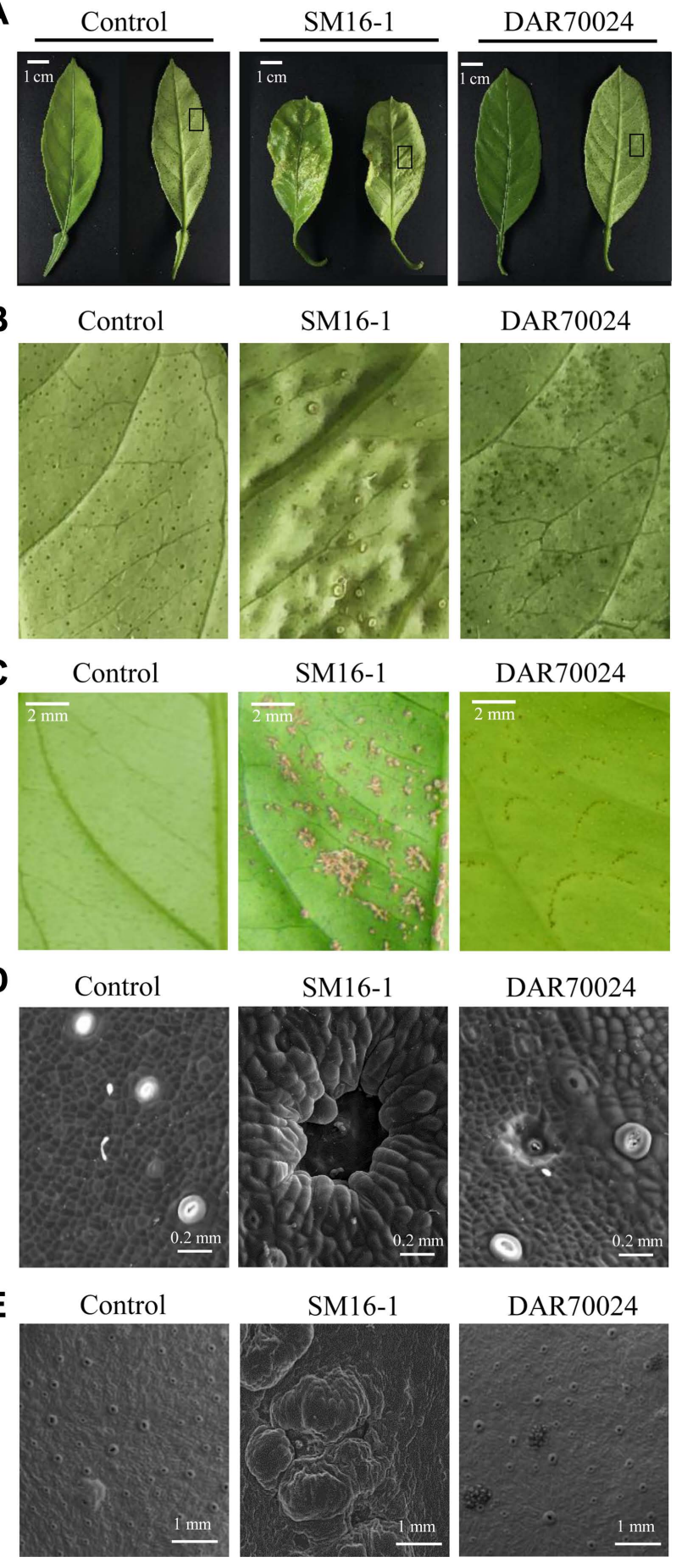

SM16-1

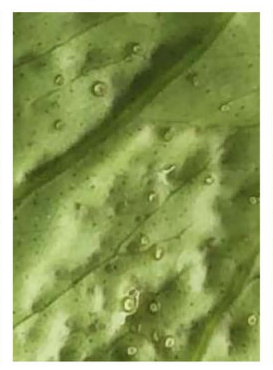

DAR70024

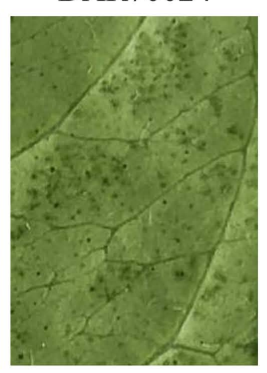

SM16-1

DAR70024

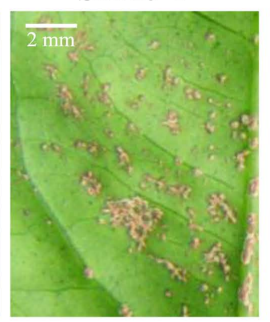

SM16-1

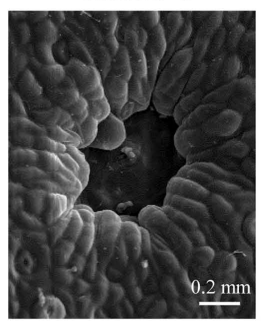

DAR70024

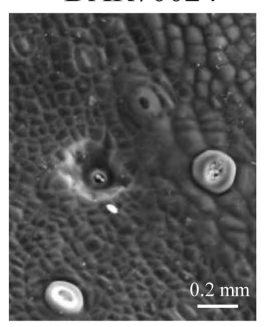

DAR70024

Fig. 1. Scab disease symptoms on satsuma mandarin leaves inoculated with water (control), a host-compatible isolate (SM16-1), and a host-incompatible isolate (DAR70024). Different symptoms were exhibited on the satsuma mandarin leaves inoculated with SM16-1 or DAR70024. The photographs were taken at 6 dpi (A) and 14 dpi (C). (B) Close-up view of black squares in (A). Initial symptoms at 1-2 dpi (D) and advanced symptoms at 5 dpi (E) in the infection site, as observed under a scanning electron microscope. 
Table 2. Elsinoë fawcettii isolates used in this study

\begin{tabular}{|c|c|c|c|c|c|c|c|c|c|c|c|}
\hline \multirow{2}{*}{ Isolate } & \multirow{2}{*}{ Host $^{\mathrm{a}}$} & \multicolumn{8}{|c|}{ Pathogenecity $^{\mathrm{b}}$} & \multirow{2}{*}{ Pathotype $^{c}$} & \multirow{2}{*}{$\begin{array}{l}\text { ITS/TEF } \\
\text { genotype }\end{array}$} \\
\hline & & $\mathrm{SM}$ & $\mathrm{RL}$ & $\mathrm{SO}$ & GF & $\mathrm{CM}$ & $\mathrm{NDF}$ & JIN & Clem & & \\
\hline SM16-1 & SM & + & + & + & + & + & $\mathrm{N}$ & - & + & FBHR & A-1 \\
\hline DAR70024 & Citrus indica & - & + & - & - & + & $\mathrm{N}$ & - & + & Tryon's & $\mathrm{A} 2$ \\
\hline
\end{tabular}

${ }^{a}$ Host of origin: SM, satsuma mandarin; RL, rough lemon; SO, sour orange; GF, grapefruit; CM, cleopatra mandarin; NDF, natsudaidai fruit; Jin, Jingeul; Clem, clementine mandarin.

bymbols: +, pathogenic; -, nonpathogenic; N, not tested.

'FBHR: Florida broad host range; Tryon's: Tryon's scab (Timmer et al., 1996)

as 1 dpi. At 5 dpi, the SM16-1 infection site surrounding the tissues was enlarged and swollen, and there was rapid division of the adjoining epidermal cells. The expanded epidermal cells formed a bulged surface that partially covered the necrotic region, and this abnormal cell growth later appeared as pustules (Fig. 1B and E). However, this altered cell growth was not observed in DAR70024-inoculated leaves, and DAR70024-induced necrotic symptoms did not change or develop (Fig. 1E).

Induced JA- and SA-related gene expression during early scab infection. To determine the cause of difference in necrotrophic patterns and abnormal cell growth in the surrounding cells, the expression patterns of genes related to plant hormone response were analyzed. The qRT-PCR results revealed that the expression of the JA biosynthe-
A
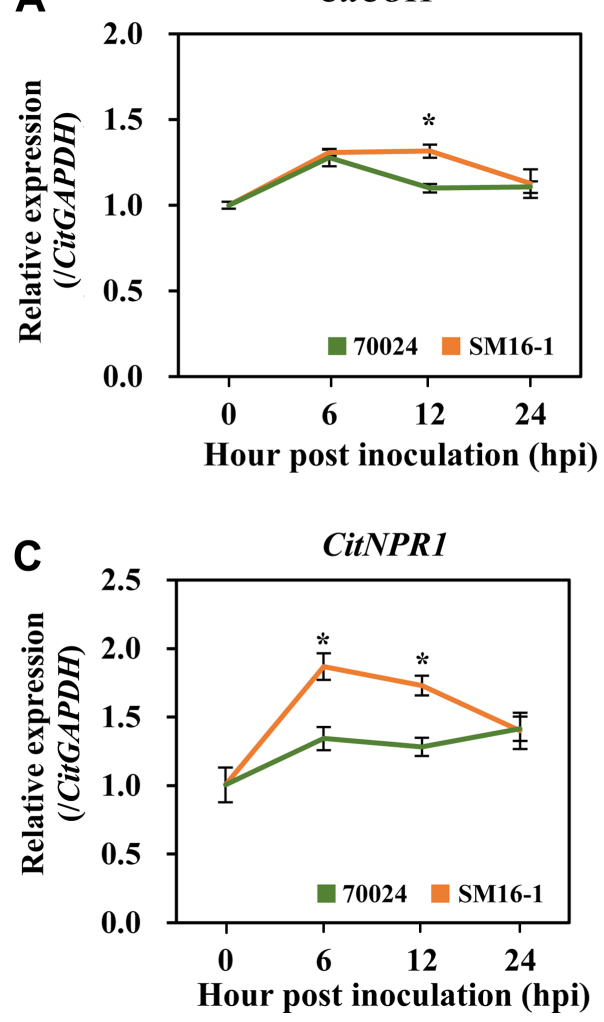

B
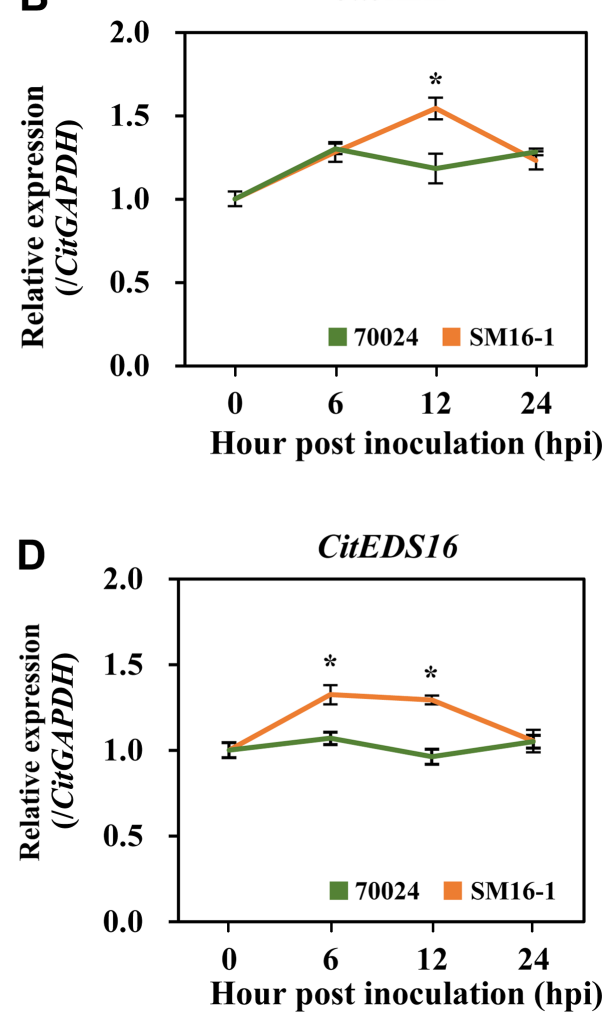

Fig. 2. Early response of salicylic acid- and jasmonic acid-responsive gene expression in satsuma mandarin leaves inoculated with a compatible isolate (SM16-1) and an incompatible isolate (DAR70024). Half of the mature leaves were inoculated and harvested for total RNA extraction at the indicated time after inoculation. Uninoculated leaves were used as the control (0 h). CitCOI1 (A), CitJAR1 (B), CitNPR1 (C), and CitEDS16 (D) were amplified with gene-specific primers pairs, and gene expression levels were calculated after normalization with the expression level of $C i t G A P D H$ and presented as values relative to that of the control $0 \mathrm{~h}$ sample. Error bars indicate the SD of the two independent experiments run in triplicates. The asterisks indicate significant differences between the sample inoculated with the compatible isolate and the incompatible isolate within the same time point, at $P<0.05$ (two-sample Student's $t$-test, $n=5$-6). 
sis gene CitCOI1 and the JA response gene CitJAR1 was induced in both SM16-1- and DAR70024-inoculated samples at $6 \mathrm{~h}$ post inoculation (hpi). Whereas the expression of CitCOI1 and CitJAR1 was continuously induced in the SM16-1-inoculated sample at $12 \mathrm{hpi}$, and that in the DAR70024-inoculated sample was reduced. Interestingly, the expression of CitJARI and CitCOII in three samples (water [as a control], SM16-1, or DAR70024) was similar at 24 hpi (Fig. 2A and B). The expression of the response and biosynthesis genes (CitNPR1 and CitEDS16, respectively) of SA, another defense-related hormone, was slightly increased in the leaves inoculated with DAR70024, but it was increased in the leaves inoculated with SM16-1 at 6 and 12 hpi. Unexpectedly, the expression of CitNPR1 and CitEDS16 was similar in the three samples at 24 dpi, and this was consistent with the expression of CitCOII and CitJAR1 (Fig. 2C and D). These results indicated that both JA and SA are involved in the defense mechanism against citrus host-compatible isolates during the early stage of infection. Moreover, the induction of SA-related gene expression was specific against the compatible isolate SM161 but not against the incompatible isolate DAR70024.

Reduced JA- and SA-related gene expression during symptom development. The initial symptoms were not observed on DAR70024-inoculated leaves, but a typical scab symptom was noted on SM16-1-inoculated leaves. We hypothesized that the hormonal responses observed in the late phase of infection, involved in symptom development, might be distinct from those observed in the
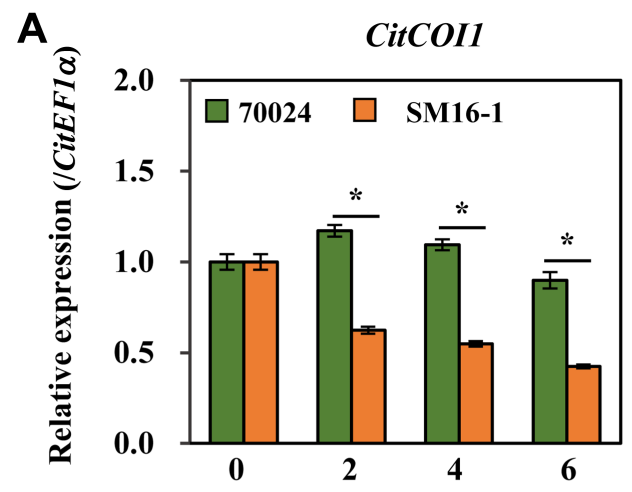

Day post inoculation (dpi)
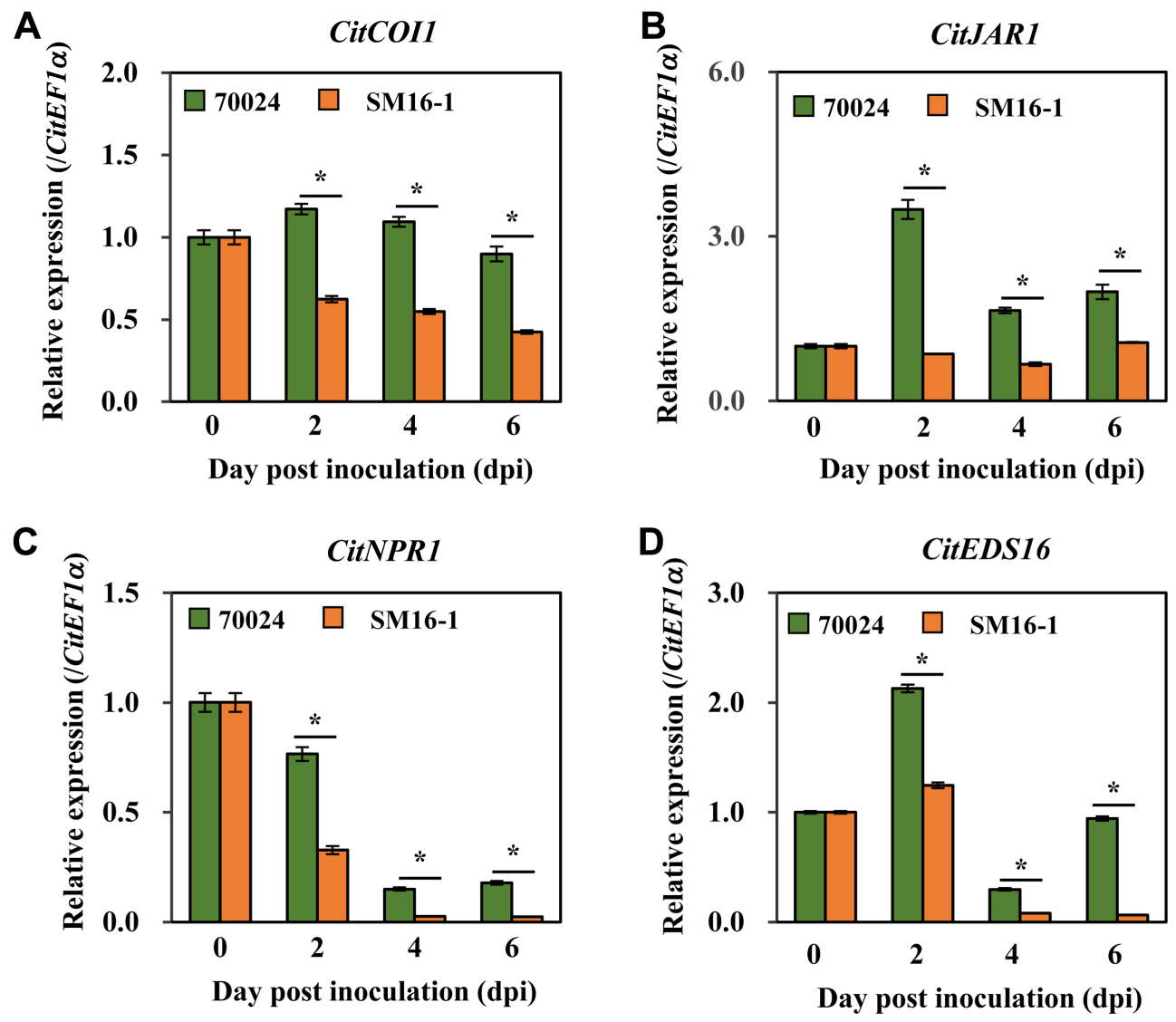

Fig. 3. Quantitative reverse-transcription polymerase chain reaction analysis of salicylic acid- and jasmonic acid-responsive gene expression levels in satsuma mandarin leaves inoculated with a compatible isolate (SM16-1) and an incompatible isolate (DAR70024) during scab symptom development. Half of the mature leaves were inoculated and harvested for total RNA extraction at the indicated time after inoculation. Uninoculated leaves were used as the control (0 dpi). CitCOI1 (A), CitJAR1 (B), CitNPR1 (C), and CitEDS16 (D) were amplified with gene-specific primers pairs, and gene expression levels were calculated after normalization with the expression level of CitEF $1 \alpha$ and presented as values relative to that of the control $0 \mathrm{~d}$ sample. Error bars indicate the SD of the two independent experiments run in triplicates. The asterisks above the bars indicate significant differences between the sample inoculated with the compatible isolate and the incompatible isolate within the same time point, at $P<0.05$ (two-sample Student's $t$-test, $n=5-6$ ). 
early phase of infection. To clarify the roles of JA and SA responses during citrus scab symptom development, we analyzed the expression of JA- and SA-related genes at 2, 4, and 6 dpi. Unexpectedly, the expression of CitNPR1, CitEDS16, CitCOI1, and CitJAR1 was reduced in the SM16-1-inoculated leaves compared with that in the DAR70024-inoculated leaves at all time points (Fig. 3). Thus, considering the qRT-PCR results in the early phase of infection, we assumed that when citrus leaves were infected with the compatible citrus scab isolate, as a defense mechanism, plants express JA- and SA-related genes. However, this induction was not maintained at 2 dpi (Fig. 3 ). Thus, citrus scab symptoms developed because of these gene expression patterns.

Induced ABA signaling during symptom development. To determine the role of ABA in citrus and E. fawcettii interaction, we analyzed the expression of ABA-response genes and ABA level at 2, 4, and 6 dpi. CitNCED3 and CitABF4 expression and ABA production were induced in both SM16-1- and DAR70024-inoculated samples. Moreover, in the SM16-1-inoculated sample, CitNCED3 and CitABF4 expression and ABA level were more significantly induced (Fig. 4). These results suggest that ABA has a positive role in citrus scab symptom development.

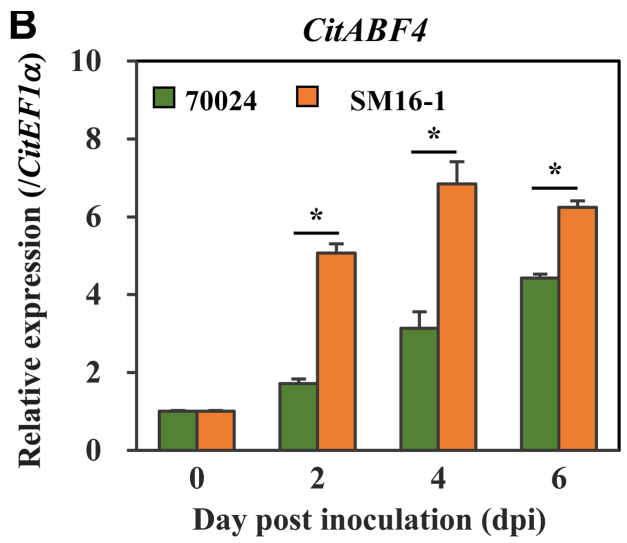

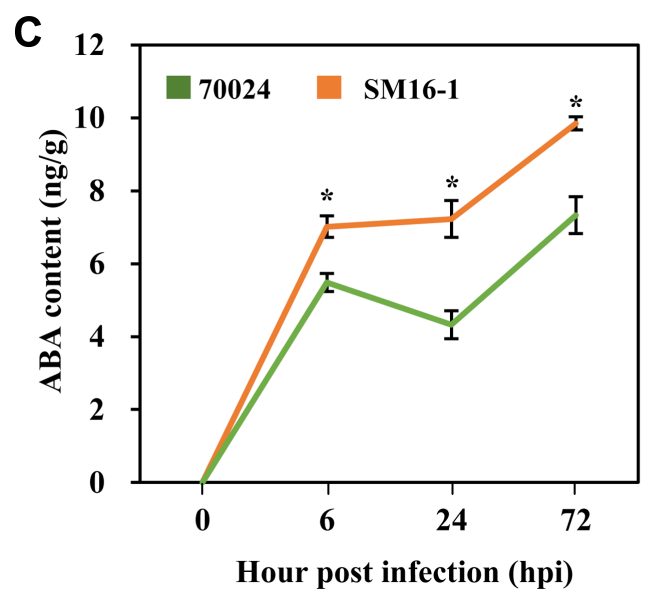

Fig. 4. Abscisic acid (ABA) response during scab symptom development. (A, B) Quantitative reverse-transcription polymerase chain reaction analysis of abscisic acid biosynthesis gene expressions in satsuma mandarin leaves inoculated with a compatible isolate (SM16-1) and an incompatible isolate (DAR70024). Uninoculated leaves were used as the control (0 dpi). CitNCED1 (A) and CitABF4 (B) were amplified with gene-specific primers pairs, and gene expression levels were calculated after normalization with the expression level of CitEF $1 \alpha$ and presented as values relative to that of the control $0 \mathrm{~d}$ sample. Error bars indicate the SD of the two independent experiments run in triplicate. The asterisks above the bars indicates significant differences between the sample inoculated with the compatible isolate and the incompatible isolate within the same time point, at $P<0.05$ (two-sample Student's $t$-test, $n=5-6$ ). (C) Levels of abscisic acid in satsuma mandarin leaves inoculated with a compatible isolate (SM16-1) and incompatible isolate (DAR70024). Half of the mature leaves were inoculated and harvested for hormone content measurement at the indicated time after inoculation. Uninoculated leaves were used as the control (0 hpi). The asterisks above the bars indicates significant differences in the samples inoculated with the compatible isolate between the incompatible isolate within the same time point at, $P<0.05$ (two-sample Student's $t$-test, $n=3$ ). 
Repressed GA signaling during scab symptom development. At 3 dpi of SM16-1 inoculation, citrus scab symptoms were visible to the naked eye. At this point, the morphology of the host epidermal cells around the growing conidia began to change, and the peripheral cells of the degraded cuticle layer were enlarged and swollen. The adjacent epidermal cells rapidly grew and enveloped the necrotic surface, including the inner germinating conidia (Chung, 2011; Paudyal et al., 2017). However, in the DAR70024-inoculated sample, there were no morphological changes after the initial symptom development. At 5 dpi, the SM16-1-inoculated sample showed typical symptoms of citrus scab, such as scabby pustules (Fig. 1A and E) and abnormal leaf growth, including smaller leaves than those in the water-treated sample and DAR70024-inoculated sample (Fig. 1A).

To investigate whether the growth regulator GA is involved in symptom development, we examined the gene expression patterns and GA level in the SM16-1- and DAR70024-inoculated samples. As expected, GA2ox2 expression was induced and GA20ox2 expression was repressed in both samples at 2, 4, and 6 dpi compared with
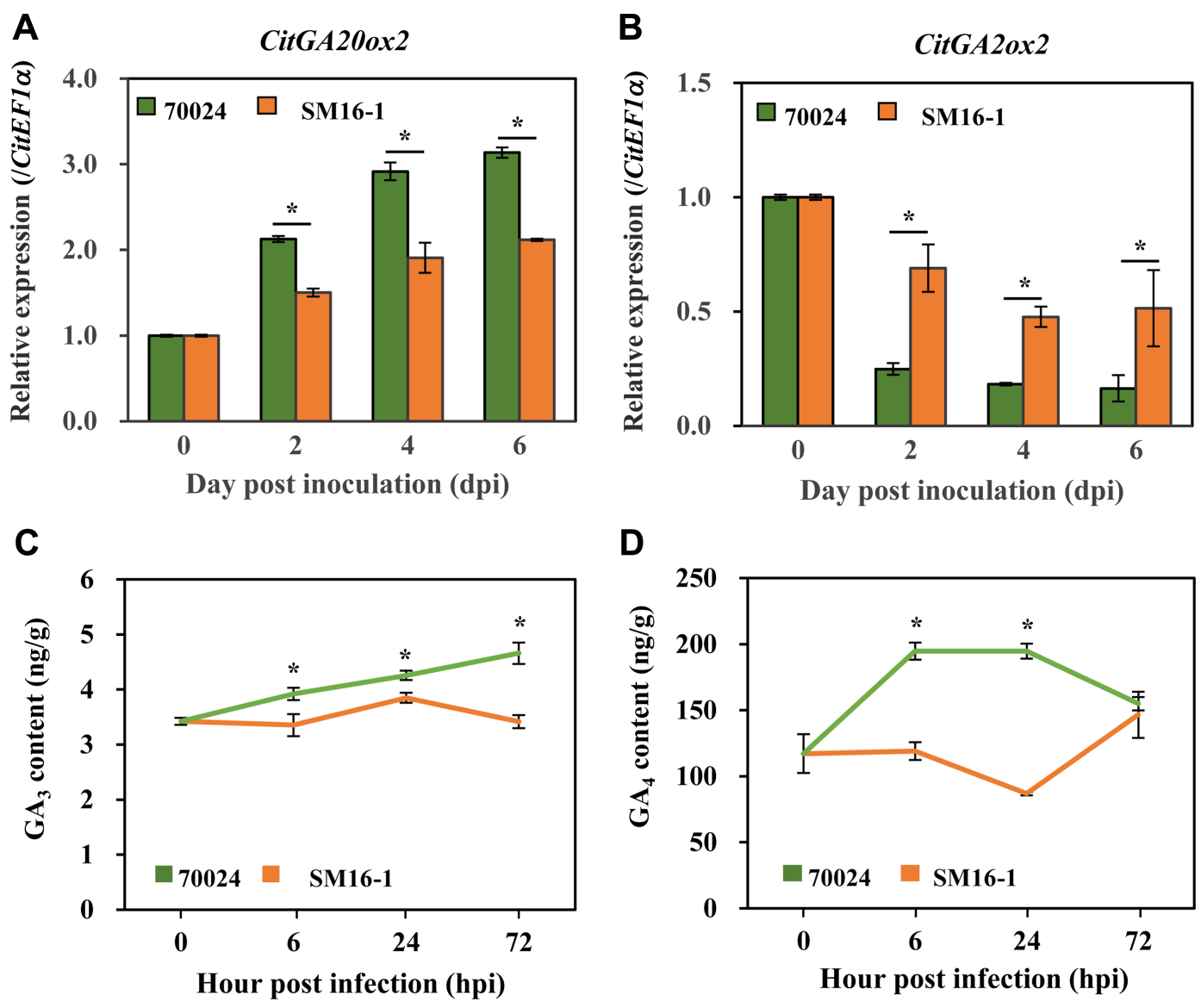

Fig. 5. Gibberellic acid (GA) response during scab symptom development. (A, B) Quantitative reverse-transcription polymerase chain reaction analysis of GA biosynthesis gene expression in satsuma mandarin leaves inoculated with a compatible (SM16-1) isolate and incompatible isolate (DAR70024). Half of the mature leaves were inoculated and harvested for total RNA extraction at the indicated time after inoculation. Uninoculated leaves were used as the control (0 dpi). CitGA2ox2 (A) and CitGA20ox2 (B) were amplified with genespecific primers pairs, and gene expression levels were calculated after normalization with the expression level of CitEF1 $\alpha$ and presented as values relative to that of the control $0 \mathrm{~d}$ sample. Error bars indicate the s.d. of the two independent experiments run in triplicates. The asterisks above the bars indicate significant differences between the sample inoculated with the compatible isolate versus the incompatible isolate within the same time point, at $P<0.05$ (two-sample Student's $t$-test, $n=5-6$ ). (C, D) Levels of GA in satsuma mandarin leaves inoculated with a compatible isolate (SM16-1) and incompatible isolate (DAR70024). Half of the mature leaves were inoculated and harvested for hormone content measurement at the indicated time after inoculation. Uninoculated leaves were used as the control ( 0 hpi). The asterisks indicate significant differences between the sample inoculated with the compatible isolate and the incompatible isolate within the same time point, at $P<0.05$ (two-sample Student's $t$-test, $n=3$ ). 
that at 0 dpi. However, in the SM16-1-inoculated sample at all investigated time points, the expression of GA2ox2 was significantly lower than that in the DAR70024-inoculated sample and the expression of GA20ox2 was significantly higher than that in the DAR70024-inoculated sample (Fig. 5A and B). In the DAR70024-inoculated sample, the GA content increased after inoculation. However, in the SM161-inoculated sample, there was no notable change in the GA level (Fig. 5C and D). Taken together, these results indicated that the GA biosynthesis pathway was repressed upon infection with the compatible $E$. fawcettii isolate.

\section{Discussion}

Plant hormones play important roles in diverse growth and developmental processes, as well as in various biotic and abiotic stress responses of plants. Infection of plants with various pathogens results in changes in the levels of various phytohormones. Here, to the best of our knowledge, we first report the hormonal response of satsuma mandarin $(C$. unshiu Marc) to E. fawcettii infection using a compatible isolate, SM16-1, and an incompatible isolate, DAR70024. The microscopy analysis showed the degradation of the protective layer and necrosis at 1-2 dpi (Fig. 1D). The process of symptom development on the leaves inoculated with SM16-1 started with enlargement, swelling, and rapid division of the adjoining epidermal cells, as previously described (Paudyal et al., 2017). However, the DAR70024inoculated leaves showed only small and shallow necrotic regions. While visible symptoms were induced at 3 dpi following SM16-1 infection (Paudyal and Hyun, 2015), no obvious altered cell growth or visible symptoms were observed in the DAR70024-infected leaves (Fig. 1E). Thus, we hypothesized that $C$. unshiu responds differently to the two isolates as early as 1 dpi.

When plants are exposed to pathogens, defense mediated by early basal defense machinery is required to prevent further fungal growth. It has also been well established that two plant defense-related hormones, JA and SA, play major roles in the plant defense machinery, and the susceptibility or tolerance of host plants to disease can be determined by these hormones. The gene expression analysis showed that in the early infection stage, both JA- and SA-related genes were induced. In particular, the SA-related genes CitNPRI and CitEDS16 were induced as early as 6 hpi. These results indicated that the initial contact with the compatible isolate of E. fawcettii rapidly triggered signal transduction to induce the JA- and SA-induced defense system. Further studies are required to examine whether the signal that induces JA and SA synthesis is mediated by a biochemical effector released from E. fawcettii or by physical contact with $E$. fawcettii. However, these inductions were not sustained for long, and the expression levels of all JA- and SA-related genes were similar at $24 \mathrm{hpi}$ and were reduced at 2, 4, and 6 dpi, as the symptoms developed (Figs. 2-4). At 5 dpi, the leaves inoculated with the compatible isolate SM161 showed severe abnormal cell growth in the tissue surrounding the infection site. However, the infection site of leaves inoculated with the incompatible isolate DAR70024 showed similar symptoms but at 1 dpi (Fig. 1E). These results indicate changes in active hormonal gene expression at 2 dpi for development of citrus scab symptoms, including altered cell growth. Thus, we conducted GA- and ABA-related gene expression analysis at 2, 4, and 6 dpi to monitor the changes in these genes during symptom development.

$\mathrm{ABA}$ has multiple functions in plants, such as responses to biotic and abiotic stresses and regulation of developmental processes (Fujita et al., 2006; Wasilewska et al., 2008). Although it is well known that ABA might have a negative role in disease resistance, its regulatory role in response to fungal diseases has also been reported, such as in tomato resistance to the necrotrophic fungus Botrytis cinerea (Audenaert et al., 2002). Several studies have also shown that ABA promotes post-invasive immunity in some cases (De Vleesschauwer et al., 2010; Hok et al., 2014). In postinvasive immunity, ABA antagonistically regulates the SA biosynthesis and signaling pathways in several plant species (Audenaert et al., 2002; Ueno et al., 2015; Yasuda et al., 2008). It has also been suggested that the positive and negative roles of ABA depend on specific plant-pathogen interactions (Mauch-Mani and Mauch, 2005; Ton et al., 2009). In this study, we showed that ABA-related genes and $\mathrm{ABA}$ production were induced during symptom development in satsuma mandarin leaves that were inoculated with the compatible E. fawcettii isolate, unlike those in the leaves inoculated with the incompatible isolate (Fig. 4). However, the SA- and JA-related genes were downregulated during symptom development but not during the early invasion stage (Figs. 2 and 3). The findings of this study suggest the possibility of an antagonistic interaction between $\mathrm{ABA}$ and $\mathrm{SA}$ in post-invasive immunity against E. fawcettii. However, future studies should validate this hypothesis with more investigations using exogenous hormone or hormone biosynthesis inhibitor treatment assays.

GA was originally identified in studies on bakanae or "foolish seedling" disease in rice. The foolish seedling disease is caused by the fungus Gibberella fujikuroi; it results in excessive seedling elongation and infertility (Stowe and Yamaki, 1957). Moreover, elevated GA level has been 
suggested to have a positive effect on nodule development after bacterial infection (McAdam et al., 2018). Although the role of GA has been clarified in response to several biotic stresses, its role in fungal infection-induced nodule development has not been established yet.

Unlike fungus-induced nodules, E. fawcettii-induced pustules are directional. On the infected side of the leaves, papillae or projections are formed, whereas the corresponding indentations form on the other side of the leaves. Here, we report the reduction in GA biosynthesis-related gene expression and endogenous GA level after compatible $E$. fawcettii isolate inoculation during pustule development. Our results indicate that there might be other unknown roles of GA involved in pustule development, different from nodule development. These results also suggest that other growth hormones, such as auxins, cytokines, and brassinosteroids, might be involved in pustule development in response to E. fawcettii infection.

Although it is well known that other fungal infections can alter physiological, metabolic, and biochemical processes (He et al., 2018; Wu et al., 2020), the mechanism by which the symptoms of abnormal tissue growth develop in the infection site is not well established. The symptom of citrus scab, a cone-like raised pustule, is a unique feature, whereas a scabby surface is a relatively common symptom of infections caused by most fungi. Thus, it is not known whether the plant itself is using a defense mechanism or manipulating the fungi, but this is an extremely interesting research topic. To the best of our knowledge, our study is the first to report the involvement of ABA and GA in citrus scab symptom development. Recently, high-quality draft genome sequences of $E$. fawcettii have been reported (Shanmugam et al., 2020), and they are expected to provide valuable information regarding the genomic attributes for determining host range through comparative genomic analyses of citrus scab fungi. Here, we reported hormonal response changes during early- and late-phase infections, during which the symptoms developed. However, further studies are required to determine the roles of SA, JA, GA, and ABA.

\section{Conflicts of Interest}

No potential conflict of interest relevant to this article was reported.

\section{Acknowledgments}

This study was supported by Project No. PJ01440101 for horticultural science and technological developments by the National Institute of Horticultural and Herbal Science, Rural Development Administration, Republic of Korea.

\section{References}

Abdelkareem, A., Thagun, C., Nakayasu, M., Mizutani, M., Hashimoto, T. and Shoji, T. 2017. Jasmonate-induced biosynthesis of steroidal glycoalkaloids depends on COI1 proteins in tomato. Biochem. Biophys. Res. Commun. 489:206-210.

Antico, C. J., Colon, C., Banks, T. and Ramonell, K. M. 2012. Insights into the role of jasmonic acid-mediated defenses against necrotrophic and biotrophic fungal pathogens. Front. Biol. 7:48-56.

Audenaert, K., De Meyer, G. B. and Höfte, M. M. 2002. Abscisic acid determines basal susceptibility of tomato to Botrytis cinerea and suppresses salicylic acid-dependent signaling mechanisms. Plant Physiol. 128:491-501.

Berens, M. L., Berry, H. M., Mine, A., Argueso, C. T. and Tsuda, K. 2017. Evolution of hormone signaling networks in plant defense. Annu. Rev. Phytopathol. 55:401-425.

Bitancourt, A. A. and Jenkins, A. E. 1936. Elsinoe fawcettii, the perfect stage of the citrus scab fungus. Phytopathology 26:393-395.

Carvalho, K., de Campos, M. K. F., Pereira, L. F. P. and Vieira, L. G. E. 2010. Reference gene selection for real-time quantitative polymerase chain reaction normalization in "Swingle" citrumelo under drought stress. Anal. Biochem. 402:197-199.

Chung, K.-R. 2011. Elsinoë fawcettii and Elsinoë australis: the fungal pathogens causing citrus scab. Mol. Plant Pathol. 12:123-135.

De Vleesschauwer, D., Yang, Y., Cruz, C. V. and Höfte, M. 2010. Abscisic acid-induced resistance against the brown spot pathogen Cochliobolus miyabeanus in rice involves MAP kinase-mediated repression of ethylene signaling. Plant Physiol. 152:2036-2052.

Dewdney, J., Reuber, T. L., Wildermuth, M. C., Devoto, A., Cui, J., Stutius, L. M., Drummond, E. P. and Ausubel, F. M. 2000. Three unique mutants of Arabidopsis identify eds loci required for limiting growth of a biotrophic fungal pathogen. Plant J. 24:205-218.

Fan, W. and Dong, X. 2002. In vivo interaction between NPR1 and transcription factor TGA2 leads to salicylic acid-mediated gene activation in Arabidopsis. Plant Cell 14:1377-1389.

Finkelstein, R. R., Gampala, S. S. and Rock, C. D. 2002. Abscisic acid signaling in seeds and seedlings. Plant Cell 14 Suppl:S15-S45.

Fujita, M., Fujita, Y., Noutoshi, Y., Takahashi, F., Narusaka, Y., Yamaguchi-Shinozaki, K. and Shinozaki, K. 2006. Crosstalk between abiotic and biotic stress responses: a current view from the points of convergence in the stress signaling networks. Curr. Opin. Plant Biol. 9:436-442.

Gopal, K., Govindarajulu, B., Ramana, K. T. V., Kishore Kumar, C. S., Gopi, V., Gouri Sankar, T., Mukunda Lakshmi, L., Naga Lakshmi, T. and Sarada, G. 2014. Citrus scab (Elsinoe 
fawcettii): a review. Res. Rev. J. Agric. Allied Sci. 3:49-58.

He, Y., Han, J., Liu, R., Ding, Y., Wang, J., Sun, L., Yang, X., Zeng, Y., Wen, W., Xu, J., Zhang, H., Yan, X., Chen, Z., Gu, Z., Chen, H., Tang, H., Deng, X. and Cheng, Y. 2018. Integrated transcriptomic and metabolomic analyses of a wax deficient citrus mutant exhibiting jasmonic acid-mediated defense against fungal pathogens. Hortic. Res. 5:43.

Hok, S., Allasia, V., Andrio, E., Naessens, E., Ribes, E., Panabières, F., Attard, A., Ris, N., Clément, M., Barlet, X., Marco, Y., Grill, E., Eichmann, R., Weis, C., Hückelhoven, R., Ammon, A., Ludwig-Müller, J., Voll, L. M. and Keller, H. 2014. The receptor kinase IMPAIRED OOMYCETE SUSCEPTIBILITY1 attenuates abscisic acid responses in Arabidopsis. Plant Physiol. 166:1506-1518.

Hyun, J.-W., Paudyal, D. P. and Hwang, R.-Y. 2015. Improved method to increase conidia production from isolates of different pathotypes of citrus scab pathogen Elsinoe spp. Res. Plant Dis. 21:231-234.

Hyun, J.-W., Timmer, L. W., Lee, S.-C., Yun, S.-H., Ko, S.-W. and Kim, K.-S. 2001. Pathological characterization and molecular analysis of Elsinoe isolates causing scab diseases of citrus in Jeju Island in Korea. Plant Dis. 85:1013-1017.

Hyun, J. W., Yi, S. H., Mackenzie, S. J., Timmer, L. W., Kim, K. S., Kang, S. K., Kwon, H. M. and Lim, H. C. 2009. Pathotypes and genetic relationship of worldwide collections of Elsino $\ddot{e}$ spp. causing scab diseases of citrus. Phytopathology 99:721728.

Livak, K. J. and Schmittgen, T. D. 2001. Analysis of relative gene expression data using real-time quantitative PCR and the $2^{-\triangle \Delta C T}$ method. Methods 25:402-408.

Mauch-Mani, B. and Mauch, F. 2005. The role of abscisic acid in plant-pathogen interactions. Curr. Opin. Plant Biol. 8:409414.

Mafra, V., Kubo, K. S., Alves-Ferreira, M., Ribeiro-Alves, M., Stuart, R. M., Boava, L. P., Rodrigues, C. M. and Machado, M. A. 2012. Reference genes for accurate transcript normalization in citrus genotypes under different experimental conditions. PLOS ONE 7:e31263.

McAdam, E. L., Reid, J. B. and Foo, E. 2018. Gibberellins promote nodule organogenesis but inhibit the infection stages of nodulation. J. Exp. Bot. 69:2117-2130.

Oliveira, M. B., Junior, M. L., Grossi-de-Sá, M. F. and Petrofeza, S. 2015. Exogenous application of methyl jasmonate induces a defense response and resistance against Sclerotinia sclerotiorum in dry bean plants. J. Plant Physiol. 182:13-22.

Park, S.-Y., Fung, P., Nishimura, N., Jensen, D. R., Fujii, H., Zhao, Y., Lumba, S., Santiago, J., Rodrigues, A., Chow, T.F. F. Alfred, S. E., Bonetta, D., Finkelstein, R., Provart, N. J., Desveaux, D., Rodriguez, P. L., McCourt, P., Zhu, J.-K., Schroeder, J. I., Volkman, B. F. and Cutler, S. R. 2009. Abscisic acid inhibits type $2 \mathrm{C}$ protein phosphatases via the PYR/ PYL family of START proteins. Science 324:1068-1071.

Paudyal, D. P. and Hyun, J.-W. 2015. Physical changes in satsuma mandarin leaf after infection of Elsinoë fawcettii causing citrus scab disease. Plant Pathol. J. 31:421-427.

Paudyal, D. P., Hyun, J.-W. and Hwang, R.-Y. 2017. Infection and symptom development by citrus scab pathogen Elsinoë fawcettii on leaves of satsuma mandarin. Eur. J. Plant Pathol. 148:807-816.

Rieu, I., Ruiz-Rivero, O., Fernandez-Garcia, N., Griffiths, J., Powers, S. J., Gong, F., Linhartova, T., Eriksson, S., Nilsson, O., Thomas, S. G., Phillips, A. L. and Hedden, P. 2008. The gibberellin biosynthetic genes AtGA20ox1 and AtGA20ox2 act, partially redundantly, to promote growth and development throughout the Arabidopsis life cycle. Plant J. 53:488504.

Shanmugam, G., Jeon, J. and Hyun, J.-W. 2020. Draft genome sequences of Elsinoë fawcettii and Elsinoë australis causing scab diseases on citrus. Mol. Plant-Microbe Interact. 33:135137.

Shigenaga, A. M. and Argueso, C. T. 2016. No hormone to rule them all: interactions of plant hormones during the responses of plants to pathogens. Semin. Cell Dev. Biol. 56:174-189.

Shimizu, T., Tanizawa, Y., Mochizuki, T., Nagasaki, H., Yoshioka, T., Toyoda, A., Fujiyama, A., Kaminuma, E. and Nakamura, Y. 2017. Draft sequencing of the heterozygous diploid genome of Satsuma (Citrus unshiu Marc.) using a hybrid assembly approach. Front. Genet. 8:180.

Staswick, P. E., Tiryaki, I. and Rowe, M. L. 2002. Jasmonate response locus JARl and several related Arabidopsis genes encode enzymes of the firefly luciferase superfamily that show activity on jasmonic, salicylic, and indole-3-acetic acids in an assay for adenylation. Plant Cell 14:1405-1415.

Sun, T.-P. 2008. Gibberellin metabolism, perception and signaling pathways in Arabidopsis. Arabidopsis Book 6:e0103.

Sussmilch, F. C., Brodribb, T. J. and McAdam, S. 2017. Up-regulation of NCED3 and ABA biosynthesis occur within minutes of a decrease in leaf turgor but $A H K 1$ is not required. J. Exp. Bot. 68:2913-2918.

Stowe, B. B. and Yamaki, T. 1957. The history and physiological action of the gibberellins. Annu. Rev. Plant Physiol. 8:181216.

Timmer, L. W., Priest, M., Broadbent, P. and Tan, M.-K. 1996. Morphological and pathological characterization of species of Elsinoe causing scab diseases of citrus. Phytopathology 86:1032-1038.

Ton, J., Flors, V. and Mauch-Mani, B. 2009. The multifaceted role of ABA in disease resistance. Trends Plant Sci. 14:310317.

Ueno, Y., Yoshida, R., Kishi-Kaboshi, M., Matsushita, A., Jiang, C.-J., Goto, S., Takahashi, A., Hirochika, H. and Takatsuji, H. 2015. Abiotic stresses antagonize the rice defence pathway through the tyrosine-dephosphorylation of OsMPK6. PLoS Pathog. 11:e1005231.

Vishwakarma, K., Upadhyay, N., Kumar, N., Yadav, G., Singh, J., Mishra, R. K., Kumar, V., Verma, R., Upadhyay, R. G., Pandey, M. and Sharma, S. 2017. Abscisic acid signaling and abiotic stress tolerance in plants: a review on current knowl- 
edge and future prospects. Front. Plant Sci. 8:161.

Wasilewska, A., Vlad, F., Sirichandra, C., Redko, Y., Jammes, F., Valon, C., Frei dit Frey, N. and Leung, J. 2008. An update on abscisic acid signaling in plants and more. Mol. Plant 1:198217.

Wu, P.-C., Chen, C.-W., Choo, C. Y. L., Chen, Y.-K., Yago, J. I. and Chung, K.-R. 2020. Biotin biosynthesis affected by the NADPH oxidase and lipid metabolism is required for growth, sporulation and infectivity in the citrus fungal pathogen Alternaria alternata. Microbiol. Res. 241:126566.

Yasuda, M., Ishikawa, A., Jikumaru, Y., Seki, M., Umezawa, T.,
Asami, T., Maruyama-Nakashita, A., Kudo, T., Shinozaki, K., Yoshida, S. and Nakashita, H. 2008. Antagonistic interaction between systemic acquired resistance and the abscisic acidmediated abiotic stress response in Arabidopsis. Plant Cell 20:1678-1692.

Yoshida, T., Fujita, Y., Sayama, H., Kidokoro, S., Maruyama, K., Mizoi, J., Shinozaki, K. and Yamaguchi-Shinozaki, K. 2010. AREB1, AREB2, and ABF3 are master transcription factors that cooperatively regulate $\mathrm{ABRE}$-dependent $\mathrm{ABA}$ signaling involved in drought stress tolerance and require ABA for full activation. Plant J. 61:672-685. 